\title{
Opuntia cladode powders inhibit adipogenesis in 3 T3-F442A adipocytes and a high-fat-diet rat model by modifying metabolic parameters and favouring faecal fat excretion
}

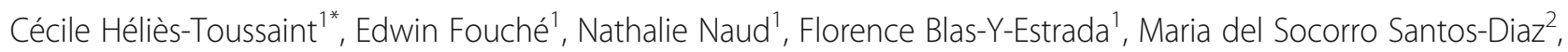
Anne Nègre-Salvayre ${ }^{3}$, Ana Paulina Barba de la Rosa ${ }^{4}$ and Françoise Guéraud ${ }^{1}$

\begin{abstract}
Background: Obesity is a major public health concern worldwide. A sedentary life and a nutritional transition to processed foods and high-calorie diets are contributing factors to obesity. The demand for nutraceutical foods, such as herbal weight-loss products, which offer the potential to counteract obesity, has consequently increased. We hypothesised that Opuntia cladodes consumption could assist weight management in an obesity prevention context.

Methods: This study was designed to explore the anti-adipogenic effects of lyophilised Opuntia cladode powders $(\mathrm{OCP})$ in an in vitro cellular model for adipocyte differentiation and an in vivo high-fat-diet (HFD)-induced obesity rat model. Two OCP were tested, one from wild species $O$. streptacantha and the second from the most known species O. ficus-indica.

Results: Pre-adipocytes 3 T3-F442A were treated by OCP during the differentiation process by insulin. OCP treatment impaired the differentiation in adipocytes, as supported by the decreased triglyceride content and a low glucose uptake, which remained comparable to that observed in undifferentiated controls, suggesting that an antiadipogenic effect was exerted by OCP. Sprague-Dawley rats were fed with a normal or HFD, supplemented or not with OCP for 8 weeks. OCP treatment slightly reduced body weight gain, liver and abdominal fat weights, improved some obesity-related metabolic parameters and increased triglyceride excretion in the faeces. Taken together, these results showed that OCP might contribute to reduce adipogenesis and fat storage in a HFD context, notably by promoting the faecal excretion of fats.
\end{abstract}

Conclusions: Opuntia cladodes may be used as a dietary supplement or potential therapeutic agent in diet-based therapies for weight management to prevent obesity.

Keywords: Opuntia, Anti-obesity, 3T3-F442A adipocytes, Rat high-fat diet, Faecal fat excretion

\footnotetext{
*Correspondence: cecile.helies@inra.fr

'INRA, ToxAlim (Research Centre in Food Toxicology), Université de Toulouse,

INRA, ENVT, INP-Purpan, UPS, Toulouse, France

Full list of author information is available at the end of the article
}

(c) The Author(s). 2020 Open Access This article is distributed under the terms of the Creative Commons Attribution 4.0 International License (http://creativecommons.org/licenses/by/4.0/), which permits unrestricted use, distribution, and reproduction in any medium, provided you give appropriate credit to the original author(s) and the source, provide a link to the Creative Commons license, and indicate if changes were made. The Creative Commons Public Domain Dedication waiver (http://creativecommons.org/publicdomain/zero/1.0/) applies to the data made available in this article, unless otherwise stated. 


\section{Background}

Obesity is a serious health issue of global concern that not only decreases life span but is also associated with the development of many chronic illnesses, such as cardiovascular diseases, type 2 diabetes, hypertension, fatty liver disease and cancer. It is considered the fifth risk of death worldwide $[1,2]$.

In 2017, Mexico had the highest global prevalence of obesity in the adult population (15-74 years, 32.4\%), while countries like Japan or Korea had the lowest incidence (below 6\%). Accelerated urbanisation, the improvement in socioeconomic conditions, and dietary changes are considered responsible for the dramatic and rapid increase in obesity prevalence. Traditional food consumption has decreased in favour of increased consumption of refined foods, as well as sweets and fat/sugar-rich diets [3]. With obesity rates projected to increase further by 2030, new strategies and cost-effective actions are needed for the prevention and control of obesity in children, adolescents and adults [4]. Promoting healthy lifestyles in the population is a major challenge for the health sector. Comprehensive public policies aimed at reversing the trend worldwide are required, particularly in Mexico.

Current anti-obesity agents often present disadvantages, while disappointing results may be observed after the arrest of lifestyle modification or pharmacotherapy, indicating a need for alternative treatment modalities that produce better and long-term results of obesity prevention or weight management [5]. Thus, identifying efficient and easy-to-use agents is a priority for medical research. Herbal supplements and diet-based therapies for weight loss are among the most common, complementary and alternative therapeutic modalities [6-8]. In Mexico, numerous plants have been identified and used in folk medicine to prevent and cure chronic diseases. Among them, Opuntia spp., including the fruit, stems, seeds and cladodes, exhibit diverse health benefits and high biotechnological potential. Opuntia cladodes are a good source of dietary fibers, which contributes to reducing body weight [5, 9-11], and the presence of antioxidants could be responsible for the nutritional and protective benefits of Opuntia-enriched diets in chronic diseases [6]. Opuntia is a species of cactus native to Mexico. Besides its medicinal purposes, it has been domesticated or naturally selected for food and ornamental use [6]. Scarce information is available concerning the effects of domestication on the biological properties of Opuntia, at the molecular and biochemical levels. In previous studies, we highlighted the variations in chemical composition and the anti-atherogenic and anti-tumoral properties of various wild and domesticated Opuntia varieties [12-14], including O. streptacantha (OSC), the wildest variety, followed by $O$. hyptiacantha, O. megacantha, O. albicarpaand O. ficusindica (OFI), which is the most known and cultivated species, with the highest degree of domestication.
Rodent studies have shown that Opuntia extracts modify obesity biomarkers. In Zucker obese rats, Nopal consumption attenuated hepatic steatosis related to obesity and reduced obesity-related metabolic abnormalities. Vinegar or isolated molecules present in Opuntia cladodes, such as kaempferol or isorhamnetin, used in obese mice models, corroborated the anti-obesity and anti-diabetic potentials of these molecules [15-21]. These studies suggest that remarkable effects could be observed in rat/mouse models of obesity induced by a high-fat diet (HFD), supplemented with Opuntia cladode powders (OCP). Further results with animal models are required to understand the underlying molecular mechanisms for these effects.

Adipose tissue growth occurs because of an increase in the size of existing adipocytes or the number of adipocytes. An imbalance between energy intake and energy expenditure generates an excess in adipose tissue resulting in obesity. Differentiation of pre-adipocytes into adipocytes involves a comprehensive network involving transcription factors responsible for the expression of key proteins that induce mature adipocyte formation. Adipogenesis also involves changes in cell morphology, induction of insulin sensitivity, and changes in $\beta$-cell secretory capacity. Deciphering the mechanism of how certain nutrients affect adipocyte differentiation and adipogenesis is important for the prevention of obesity and related diseases [22].

The present study was designed to investigate whether Opuntia species exert anti-obesity properties by examining the anti-adipogenic effect of two Opuntia cladode powders (OCP) and elucidating the mechanisms underlying such effects. For this purpose, we used powders from the wildest (OSC) and the most domesticated (OFI) Opuntia varieties. We chose these two species for their highest domestication gradient difference (from the ancestor specie (OSC, growing in wild habitat) to the most propagated Opuntia for commercial production (OFI). The differentiation of pre-adipocytes into adipocytes was examined by treating 3 T3-F442A cells [22] with OCP to investigate the effects at the cellular level. The same powders were also tested on an animal model of obesity by feeding Sprague-Dawley rats with a highfat diet (HFD) supplemented or not with the powders. This animal model allowed us to examine the wholebody level, as the first step towards human trials.

\section{Methods \\ Reagents}

Cytochalasin B, 2-deoxyglucose, insulin and Dulbecco's modified Eagle's medium (DMEM) were all purchased from Sigma-Aldrich (Saint-Quentin-Fallavier, France). $\left[{ }^{3} \mathrm{H}\right]$-2-deoxyglucose was from PerkinElmer (Boston; WalthamMA, USA). 
Opuntia plant material and cladode powder preparation Opuntia young cladodes from the wild species OSC $(O$. streptacantha Lem., cv. Tuna Loca) and OFI (O. ficusindica [L.] Mill., cv. RojoVigor) were collected (April 2010 and 2012) from the Opuntia Germplasm Bank of the Agrobotanical Garden located in El Orito, Zacatecas, Mexico. The formal identification of the plants was reported by Ramirez-Tobias et al. [23]. The plants were grown under the same environmental conditions [12]. Cladodes were washed, ground in liquid nitrogen using a KrupsGX 4100 grinder (Mexico City, Mexico), and kept at $-80^{\circ} \mathrm{C}$ until further processing. Samples were freezedried (Labconco, Kansas City, MO, USA), sieved through mesh 80 , then stored in plastic bags at $4{ }^{\circ} \mathrm{C}$ until use [14]. Contents of fat, crude fiber and total phenolic compounds were determined, as previously reported [12].

\section{Cell culture and adipocyte differentiation Cell culture}

The 3 T3-F442A cell line was a gift from Prof. P. Valet (I2MC, Toulouse, France). Pre-adipocytes were cultured in DMEM with penicillin $(100 \mathrm{UI} / \mathrm{mL})$-streptomycin $(0.1 \mathrm{mg} /$ $\mathrm{mL}$ ) (Sigma-Aldrich, Saint Quentin Fallavier; France) supplemented with $10 \%$ foetal calf serum (Gold Serum, PAA Laboratories, Les Mureaux, France). Cells were cultured at $37^{\circ} \mathrm{C}$ in a $5 \% \mathrm{CO}_{2}$ humidified atmosphere. Differentiation was induced by incubating confluent 3 T3-F442A cells in a differentiation medium (DMEM supplemented with 10\% foetal calf serum and $50 \mathrm{nM}$ insulin) for up to 10 days, with the culture medium renewed every $2-3$ days. The noninsulin-treated cells were considered as non-differentiated controls. Treatments with Opuntia powders, prepared as described above, were applied during the 10 days of differentiation, concomitantly with insulin treatmentFor all in vitro experiments, the two OCP were diluted directly in the culture medium $(100 \mu \mathrm{g} / \mathrm{mL})$, mixed vigorously, and filtered at $0.2 \mu \mathrm{M}$ before dilution in DMEM to 1,10 and $100 \mu \mathrm{g} / \mathrm{mL}$. The culture medium was changed every $48 \mathrm{~h}$, renewing OCP at the corresponding concentrations in the medium. Cells were seeded at $7.5 \times 10^{4}$ cells/well in 6-well plates for triglyceride (TG) content evaluation, at $2 \times 10^{4}$ cells/well in 24well plates for glucose uptake assays and at $5 \times 10^{3}$ cells/well in 96-well plates for cytotoxicity (3-[4,5-dimethylthiazol-2yl]-2,5-diphenyltetrazolium bromide, MTT).

Triglyceride (TG) assay The intracellular TG was quantified using the TG PAP 150 enzymatic kit (TG PAP 150, BioMérieux, Marcy l'Etoile, France) after cell lysis $(0.1 \mathrm{~N}$ $\mathrm{NaOH})$. To account for cellular proliferation or toxicity of the molecules, the TG content was related to the protein content, determined by the bicinchoninic acid (BCA) enzymatic kit (Pierce, Thermofischer Scientific, Bordeaux, France). The results were expressed as the percentage of control cells treated only with insulin $(50 \mathrm{nM})$.
Glucose uptake assay Glucose uptake was measured according to Kim et al. [24] with some modifications [25]. Briefly, after 10 days of treatment, cells were washed twice in serum-free DMEM and pre-incubated in this medium at $37^{\circ} \mathrm{C}$ for $16 \mathrm{~h}$. After this starvation period, cells were washed twice with Krebs-Ringer bicarbonate buffer (KRB) and incubated at $37^{\circ} \mathrm{C}$ for $30 \mathrm{~min}$ with $100 \mathrm{nM}$ insulin (or not, for the negative control). To initiate glucose uptake, 2-deoxy- $\left[1-{ }^{3} \mathrm{H}\right]$ glucose $(1 \mu \mathrm{Ci} / \mathrm{mL})$ diluted in $0.1 \mathrm{mMD}$-glucose solution was added to each well and the plates then incubated at $37^{\circ} \mathrm{C}$ for $10 \mathrm{~min}$. After incubation, the cells were washed twice with ice-cold KRB buffer and lysed in $0.1 \mathrm{~N} \mathrm{NaOH}$. Half of the content of each well was transferred to scintillation vials, and $10 \mathrm{~mL}$ of scintillation cocktail (Ultima Gold, Perkin Elmer, Boston, WalthamMA, USA) was added. The radioactivity incorporated into the cells was measured using a liquid scintillation counter (Hewlett Packard, USA). The BCA protein content was assayed for each point on the remaining half.

Determination of cellular toxicity After incubation, the wells were gently rinsed with cold phosphatebuffered saline, and then $20 \mu \mathrm{L}$ of $5 \mathrm{mg} / \mathrm{mL}$ MTT was added to each well and incubated for $4 \mathrm{~h}$. Subsequently, the media from each well was gently aspirated, and $100 \mu \mathrm{L}$ of dimethylsulphoxide was added to dissolve the formazan crystals. Plates were shaken for $30 \mathrm{~min}$, followed by absorbance measurements at $570 \mathrm{~nm}$ using a Tecan microplate reader (Tecan, Raleigh, USA).

\section{Animal experiments \\ Animals and diets}

Male Sprague-Dawley rats (6-weeks-old, body weight of $200 \mathrm{~g}$ ) were purchased from Charles River Laboratory (Saint-Germain-Nuelles, France). Rats were housed in polycarbonate cages maintained at $24{ }^{\circ} \mathrm{C}$, with $40-70 \%$ humidity and 12/12- h light/dark cycles, with free access to food and water. The rats were housed in groups of two to avoid single animals. The protocol was approved by the local ethics committee (TOXCOM/0011/FG FG). A total of 40 rats were randomly distributed into four groups of 10 animals for the following treatments: control group fed with the standard diet (SD); HFD-fed group (F), and HFD-fed group supplemented with $0.5 \% \mathrm{w} / \mathrm{w}$ OCP OSC (F-OSC) and OFI (F-OFI), respectively. Cages were randomly assigned to the racks, to avoid artefacts related to the position. The experimental diets were fed for 60 days. All diets were based on a modified standard AIN76 diet, prepared and formulated in a powdered form by the Experimental Feeds Preparation Unit (UPAE, INRA, Jouy-en-Josas, France) and stored at $20{ }^{\circ} \mathrm{C}$. The ingredients and macronutrients composition of the diets $(\mathrm{g} / \mathrm{kg})$ are listed in Table 1 . The HFD was enriched with $25 \%$ lard (Cooper 1, Lamballe, France). The lipid percentages are listed in Table 2 . The diets were renewed every 2-3 days and distributed randomly. The diet intake and the 
Table 1 Ingredients and macronutrients composition of the diets $(\mathrm{g} / \mathrm{kg})$

\begin{tabular}{llll}
\hline Ingredient & SD & F & F-OFI or \\
\hline Casein & 400 & 200 & 199 \\
Corn starch & 150 & 50 & 49,7 \\
Sucrose & 298 & 348 & 346,3 \\
Cellulose & 50 & 50 & 49,7 \\
Methionin & 3 & 3 & 3 \\
Minerals & 34,8 & 34,8 & 34,6 \\
Vitamin mix (type AIN76) & 10 & 10 & 9,9 \\
Calcium phosphate & 2,1 & 2,1 & 2,1 \\
Choline bitartrate & 2 & 2 & 2 \\
Ferric citrate & 0.144 & 0.144 & 0.14 \\
Safflower oil & 50 & 50 & 49.7 \\
Lard & 0 & 250 & 248.7 \\
OCP & 0 & 0 & 5
\end{tabular}

$\mathrm{SD}=$ standard diet; $\mathrm{F}=$ high fat diet; F-OFI or F-OSC $=$ Cladode powder from $O$. ficus-indica (OFI) or $\mathrm{O}$. streptacantha $(\mathrm{OSC}) ; \mathrm{OCP}=$ Opuntia cladode powder.

animals' body weight were monitored every $2-3$ days in the morning, and animal welfare was checked simultaneously. The number of animals per group $(n=10)$ was calculated to obtain statistically significant results for diet-induced changes. The days before the end of the experiment, 24-h faeces were collected and stored at $-80^{\circ} \mathrm{C}$. At the end of the study, the rats were killed by $\mathrm{CO}_{2}$ asphyxiation according to the protocol of the French "National Charter Concerning the Ethics of Animal Experimentation". Briefly, each rat was placed in a $25-\mathrm{L}$ polycarbonate chamber. Then, $\mathrm{CO}_{2}$ was emitted into the chamber at a flow rate of about $5.5-7.5 \mathrm{~L} / \mathrm{min}$ until the rat was unconscious. The $\mathrm{CO}_{2}$ flow continued for at least $60 \mathrm{~s}$ to ensure that the breath was not seen before removing the rat from the chamber. Blood samples were collected from the inferior vena cava for biochemical analysis. Liver and abdominal fat were immediately removed, weighed and stored at $-80^{\circ} \mathrm{C}$.

Biochemical analysis Plasma samples were separated from blood cells by centrifugation at $1000 \times g$ for $15 \mathrm{~min}$. Plasma levels of leptin, insulin and monocyte chemoattractant protein-1 (MCP-1) were analysed by the Luminex kit
(RMHMAG-84 K-05) (Thermofisher, Bordeaux, France). TG, glucose and hepatic parameters (aspartate aminotransferase, AST; alanine aminotransferase, ALT) were all measured at the Anexplo Facilities, Toulouse (France). Adiponectin was evaluated using the TECO medical Mediagnost (E091-Rkit, Reutlingen, Germany), and C-reactive protein (CRP) was measured using the Abcam kit (ab108827, Abcam, Cambrigde, UK).

Measurement of hepatic and faecal triglyceride (TG) levels Hepatic lipids were extracted by homogenisation of liver tissues in phosphate-buffered saline. Ethanol was added to the homogenates $(1: 9, \mathrm{v} / \mathrm{v})$, and the samples were mixed at room temperature for $1 \mathrm{~h}$ to solubilise the TG. After centrifugation at $2000 \times g$ for $10 \mathrm{~min}$, the supernatant was collected to measure the TG level using the TG PAP 150 enzymatic kit (TG PAP 150, BioMérieux, Marcy l'Etoile, France), according to the manufacturer's protocol. Faecal TG lipids were extracted from the faeces collected over $24 \mathrm{~h}$. The samples were homogenised in sterilised water, then centrifuged at $2000 \times g$ for $10 \mathrm{~min}$. The supernatant was collected to measure the TG level using the TG PAP 150 enzymatic kit (TG PAP 150, BioMérieux, Marcy l'Etoile, France), according to the manufacturer's protocol.

\section{Statistical analysis}

All data were expressed as mean \pm standard error of the mean (SEM) of three (or more) independent experiments (cell experiments) or 10 rats per group (in vivo experiments). Statistical significance was determined by one-way analysis of variance (ANOVA), followed by Newman-Keulspost hoc test, using GraphPad Prism software. Statistical significance was indicated by ${ }^{*} p<$ $0.05,{ }^{* *} p<0.01$ and ${ }^{* * * *} p<0.005$. In Fig. $1,{ }^{\mathbb{S S}} p<0.01$, ${ }^{\mathbb{S S S}} p<0.005$ indicates significant difference between groups in insulin-treated cells. In Figs. 2 and $4{ }^{\$} p<0.05$; ${ }_{\mathbb{S S}} p<0.01$, denotes significant difference of F-OCP and F-OFI from the F group.

\section{Results}

Characterisation of biological compounds in the two Opuntia cladode powders (OCP)

For each species (OSC, OFI), young cladodes at a similar maturity stage were collected. The contents of macromolecules,

Table 2 Lipid composition in diets

\begin{tabular}{|c|c|c|c|}
\hline Fat & Saturated & Unsaturated & Other \\
\hline Safflower oil & $\begin{array}{l}10 \% \\
7 \% \text { Palmitic acid (16:0) } \\
3 \% \text { Stearic acid (18:0) }\end{array}$ & $\begin{array}{l}\text { 89\% } \\
75 \% \text { Linoleic acid }(18: 2 n-6) \\
14 \% \text { Oleic acid }(18: 1 n-9)\end{array}$ & $1 \%$ \\
\hline Lard (hog fat) & $\begin{array}{l}40 \% \\
27 \% \text { Palmitic acid (16:0) } \\
11 \% \text { Stearic acid (18:0) } \\
2 \% \text { Myristic acid (14:0) }\end{array}$ & $\begin{array}{l}59 \% \\
44 \% \text { Oleic acid } \quad(18: 1 n-9) \\
11 \% \text { linoleic acid } \quad(18: 2 n-6) \\
4 \% \text { palmitoleic acid } \quad(16: 1 n-7)\end{array}$ & $1 \%$ \\
\hline
\end{tabular}




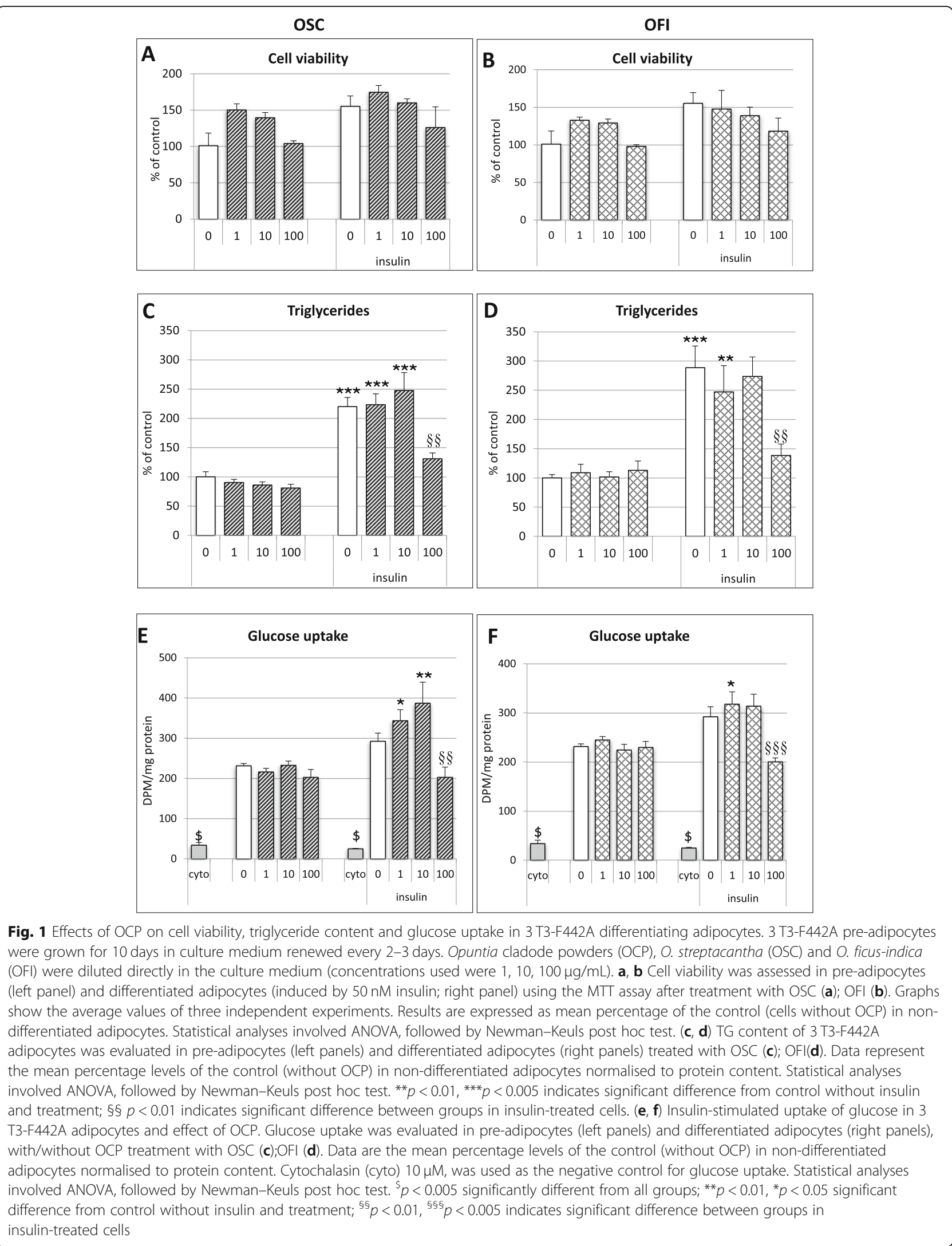




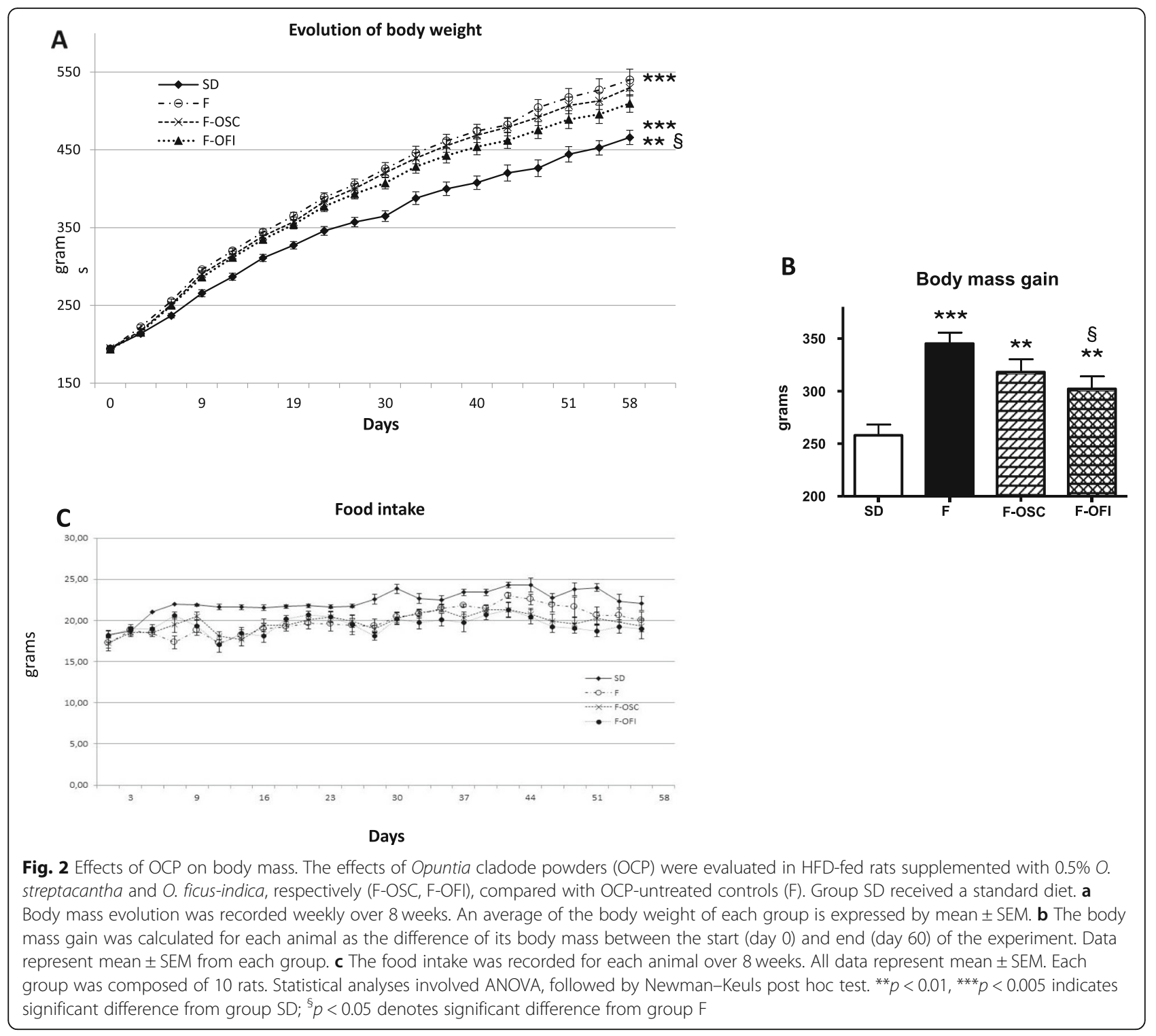

phenolic acids and flavonoids, and the antioxidant capacities of the OCP were analysed in a previous study [12]. The results are summarized in Table 3. The proximal composition showed no differences in protein (11.0 and 11.7\%) and fat content (0.62 and $0.68 \%$ ), but OSC had higher fibre content (6.52\%), while OFI presented the highest ash content (14.2\%). OSC contained the highest phenolic compound (65.1 $\mu \mathrm{g}$ gallic acid equivalent/g sample) concentration and antioxidant capacity, but no difference in flavonoid content between the two species was observed.

Effects of Opuntia cladode powders (OCP) on 3 T3-F442A pre-adipocyte differentiation

\section{Cytotoxic effects of Opuntia cladode powders (OCP)}

To evaluate the potential cytotoxic effects of OCP, preadipocytes were differentiated into mature adipocytes for
10 days with $50 \mathrm{nM}$ insulin, in the presence of various concentrations of OCP $(0,1,10$ and $100 \mu \mathrm{M})$. As shown in Fig. 1, treatment with OSC (Fig. 1a) and OFI (Fig. 1b) had no markedly effect on cell viability, around $100 \%$ of the control, even with the highest OSC concentration $(100 \mu \mathrm{M})$. At lower concentrations $(1$ and $10 \mu \mathrm{M})$, the cell viability was increased, possibly due to a mitogenic effect of OCP.

\section{Opuntia cladode powders (OCP) inhibit triglyceride (TG) storage in adipocytes}

To examine the effects of OCP on the differentiation of 3 T3-F442A pre-adipocytes in adipocytes, confluent cells were treated with increasing concentrations of OCP. As shown in Fig. 1c, d, the TG content was not affected by OSC and OFI, in undifferentiated cells (without insulin). 
Table 3 Proximal composition, phenolic compounds, and antioxidant capacity of both Opuntia streptacantha and Opuntia ficus-indica cladode powders

\begin{tabular}{|c|c|c|c|}
\hline \multirow[t]{2}{*}{ Biomolecules } & \multicolumn{2}{|l|}{ Opuntia } & \\
\hline & streptacantha & ficus-indica & \\
\hline \multicolumn{4}{|c|}{ Proximal composition (\%) $^{a}$} \\
\hline Protein & 11.0 & 11.7 & \\
\hline Fat & 0.62 & 0.68 & \\
\hline Fibre & 6.52 & 5.63 & $\$$ \\
\hline Ash & 12.6 & 14.2 & $\$$ \\
\hline Phenolic acids ${ }^{b}$ & 65.1 & 56.7 & $\$$ \\
\hline Flavonoids $^{c}$ & 19.0 & 20.4 & \\
\hline Antioxidant capacity ${ }^{d}$ & 897.8 & 659.4 & $\$$ \\
\hline
\end{tabular}

${ }^{a}$ values are the mean of triplicates on dry weight basis.

bas $\mu \mathrm{g}$ of gallic acid/g sample;

cas $\mu$ g of quercetin $/ g$ sample;

${ }^{d}$ as $\mu \mathrm{mol}$ of Trolox/g sample.

$\$$ statistically differences between means.

Differentiated cells exhibited a significant increase in intracellular TG content (about 250\%) which was significantly reduced in cells treated with $100 \mu \mathrm{M}$ OCP (OSC, 60\%; OFI, 50\%; $p<0.01)$. No effect was observed for lower OCP concentrations ( 1 and $10 \mu \mathrm{M})$.

\section{Effects of Opuntia cladode powders (OCP) on cellular uptake of glucose}

The differentiation of pre-adipocytes into mature adipocytes is characterized by a strong increase in glucose uptake in response to insulin [26]. As high OCP concentrations tend to decrease the differentiation of 3 T3-F442A cells into mature adipocytes, we checked the effect of OCP on the glucose uptake evoked by insulin in undifferentiated and differentiated cells. The results presented in Fig. 1 e,f showed that insulin stimulated the uptake of glucose under our experimental conditions. Opuntia powders had no noticeable effect on glucose uptake in the absence of insulin. When cells were differentiated by insulin, low-to-moderate concentrations of OCP slightly increased the uptake of glucose elicited by insulin. However, when cells were incubated with high OSC and OFI concentrations $(100 \mu \mathrm{g} / \mathrm{mL})$ during the differentiation process, the glucose uptake remained similar to that observed in undifferentiated $3 \mathrm{~T} 3-$ F442A cells (70\% OSC and 60\% OFI of the control cells treated with insulin but without OCP; $p<0.001$ ). Altogether, these results (low TG levels and low glucose uptake) suggested that OCP prevented or reduced the differentiation of 3 T3-F442A cells into mature adipocytes.

\section{Opuntia cladode powders (OCP) supplementation in diet prevented HFD-induced obesity Body weight and food intake}

To investigate whether OCP may modulate obesity in an animal model, Sprague-Dawley rats were HFD-fed supplemented with $0.5 \%$ OCP. The percentage of powders used in rat diets was chosen as an average of the percentage used in previous studies of 0.25 to $1 \%$ [14, 15, 22, 27]. OSC and OFI were given for 8 weeks and compared with HFD given without supplement and standard diet (SD). Growth parameters were evaluated throughout the 60 days of OCP treatment for each rat. Initial body weights were not dramatically different among the groups. After 8 weeks, the final body weights were significantly higher in the three HFD groups (F, F-OSC, F-OFI), when compared to the control (SD) group (Fig. 2a; $p<0.005$ ). HFD-fed rats supplemented with OFI (F-OFI) exhibited a body mass significantly lower $(87.5 \% ; p<0.05)$ when compared to group F (Fig. 2a). Likewise, the final body mass was lower in group F-OSC, but not significant. The body mass gain (Fig. 2b), representing the average of individual differences of body mass from the initial and final body mass, presented a significant difference between the HFD diet and standard diet SD (F; $p<0.005$, OCP; $p<0.01)$. A significantly lower gain in body mass was observed for OFIsupplemented rats when compared to the control group (F) $(p<0.05)$. No significant differences in food intake were observed between the three HFD groups throughout the experiment, indicating that the reduction in body weight gain in the OFI group was not due to reduced food intake (Fig. 2c). It is to note that the weekly food intake was higher in the SD group than for the HFD (F) groups (Fig. 2c). The weight of abdominal fat was significantly higher in HFD than SD groups $(200 \% ; p<0.005)$. Both OCP reduced this increment by about $80 \%$ between FOSC and F-OFI vs SD; $p<0.05$; (Fig. 3a). Same results were observed for liver weight (Fig. $3 \mathrm{~b}$ ), even if the slight decreases observed in groups F-OSC and F-OFI were not markedly different from group $\mathrm{F}$ and the SD group.

\section{Metabolic parameters}

The effects of OCP supplementation were investigated on metabolic parameters, by measuring the serum levels of obesity-related markers (Fig. 4). As shown in Fig. 4 a, b, the increase in adiposity was associated with a decrease in adiponectin level in group $\mathrm{F}(\mathrm{F}=75 \%$ vs SD; $p<0.01)$ and an increase in leptin level ( $\mathrm{F}=260 \%$ vs SD; $p<0.005)$. OCP supplementation in food restored the adiponectin level $\quad(\mathrm{F}-\mathrm{OSC}=143 \% ; \mathrm{p}<0.01, \mathrm{~F}-\mathrm{OFI}=126 \% ; \mathrm{p}<0.05$, compared to the $\mathrm{F}$ group). A significant increase in leptin level was observed in the 3 HFD groups (when compared to $\mathrm{SD}, \mathrm{F}-\mathrm{OSC}=195 \% ; p<0.01$ and $\mathrm{F}-\mathrm{OFI}=180 \% ; p<0.01$, as compared with $260 \%$ in $\mathrm{F} ; p<0.005$ ), with a decrease of 25 and 30\% for F-OSC and F-OFI respectively compared to the $\mathrm{F}$ - group, $p<0.05$ ).

HFD diet slightly increased the levels of circulating TG (130\%), and this was reversed by both OCP supplementation (Fig. 4c) though not significantly. Same observations could be done for circulating glucose levels. OCP supplementation 

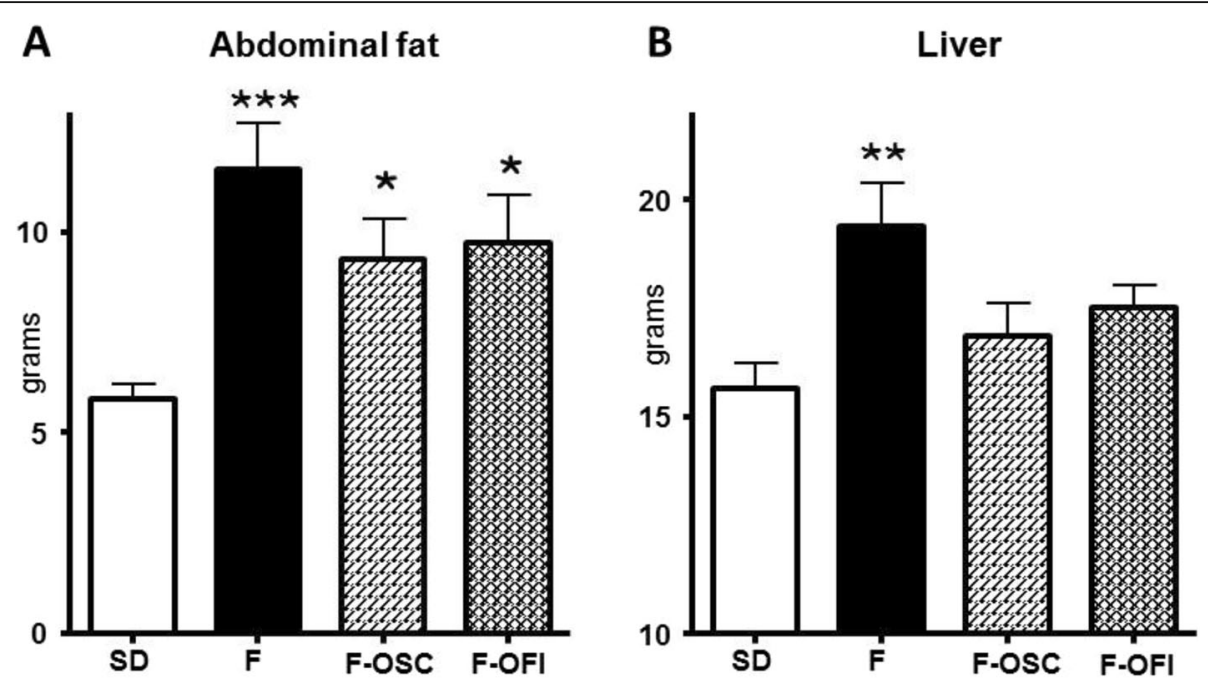

Fig. 3 Effects of Opuntia cladode powders (OCP) supplementation on relative abdominal fat mass (a) and liver mass (b). Data represent mean \pm SEM. Statistical analysis involved ANOVA, followed by Newman-Keuls post hoc test. ${ }^{*} p<0.05,{ }^{* *} p<0.01,{ }^{* * *} p<0.005$ indicates significant difference from the group fed the standard diet (SD)

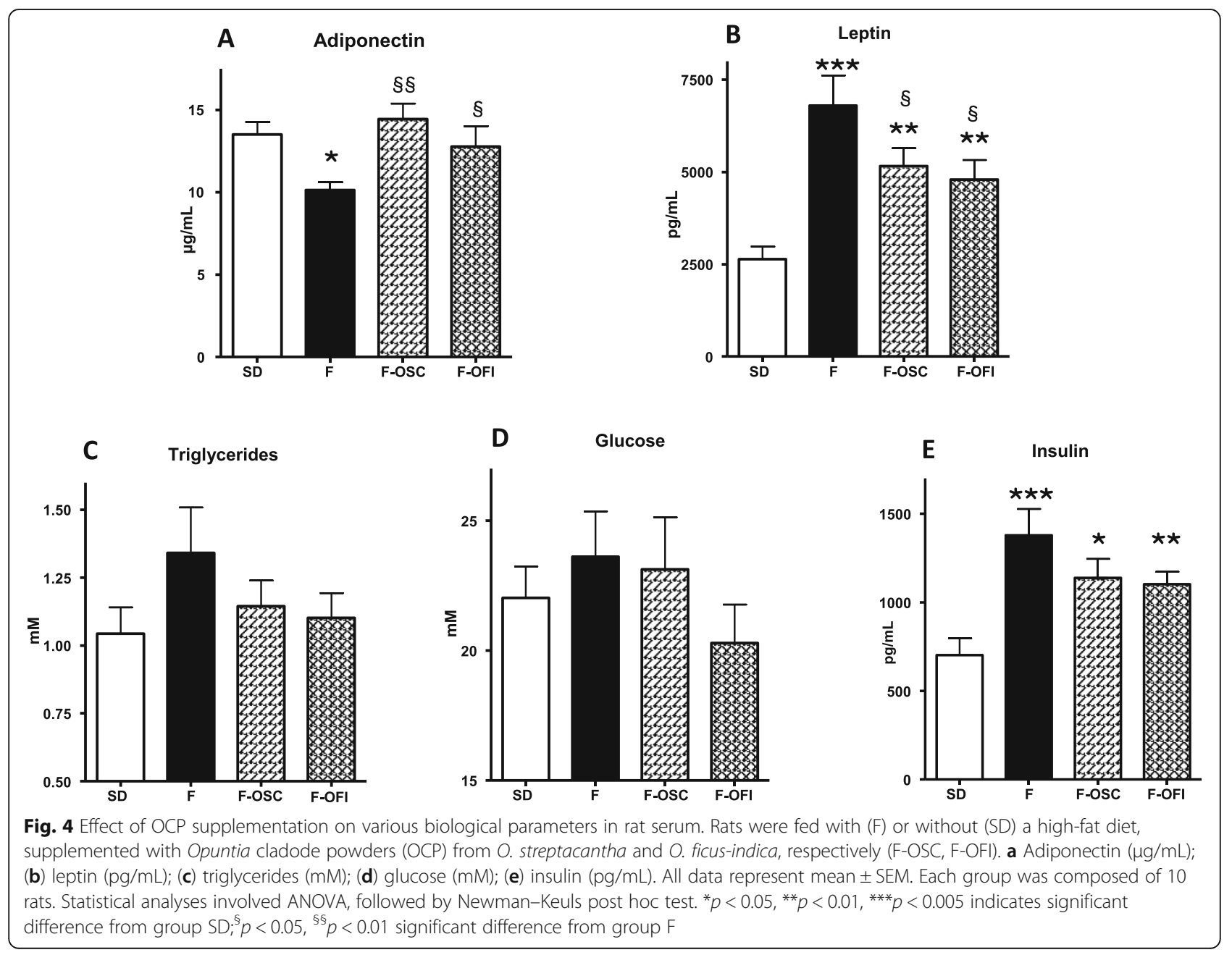


tended to decrease TG and glucose levels (Fig. 4c,d). Likewise, circulating insulin levels (Fig. 4e) were increased in group $\mathrm{F}(195 \% ; p<0.005)$ by comparison with the SD group, and were reduced by OCP supplementation (18 and $20 \%$ for F-OSC and F-OFI respectively).

\section{Effects of OCP supplementation on liver and inflammation}

HFD causes hepatic inflammation and steatosis, which can further lead to non-alcoholic steatohepatitis and non-alcoholic fatty liver disease (NAFLD) [28]. To evaluate the potential toxicity of HFD and OCP supplementation, serum markers for liver injury and liver TG content were measured in our rat model. No noticeable differences were observed among the different groups concerning the levels of AST and ALT and inflammation markers, such as MCP-1 (Table 4) and CRP (data not shown), Therefore, neither HFD nor OCP supplementations caused noticeable adverse toxic effects in rats. The hepatic TG content (Fig. 5a) was significantly increased due to HFD diet (HFD groups, $235 \%$ vs SD; $p<0.05$ ). OFI supplementation slightly reduced the TG content (85\% of group F; $p<0.05$ ), but not significantly.

\section{Triglyceride (TG) evaluation in faeces}

Our results indicated that HFD-fed rats supplemented with OCP, presented a lower body mass and reduced TG levels in the serum and the liver when compared to HFD (F) control rats. To understand the mechanisms implicated in the decreased TG levels, the TG content of rat faeces was measured 3 days before the end of experiments. The results in Fig. 5b, indicate that TG concentration was increased in faeces from the three HFD groups, particularly in the group F- OFI $(123 \% ; p<0.05)$, when compared to the SD group. However the TG content in faeces from this group was comparable to the F and F-OSC groups.

\section{Discussion}

In the present study, we investigated the effects on obesity of two OCP, the wildest OSC and the most domesticated OFI, using 3 T3-F442A adipocytes cells and HFD obese rats.

Adipocytes play a central role in the maintenance of lipid homeostasis and energy balance by storing TG or releasing free fatty acids in response to changes in

Table 4 Serum hepatic markers

\begin{tabular}{lllll}
\hline Marker & SD & F & F-OSC & F-OFI \\
\hline AST (U/L) & $32.6 \pm 2.42$ & $30.3 \pm 1.78$ & $35.0 \pm 3.26$ & $37.0 \pm 4.61$ \\
ALT(U/L) & $78.3 \pm 3.91$ & $64.6 \pm 1.99$ & $78.2 \pm 6.45$ & $72.8 \pm 4.49$ \\
MCP-1 (pg/mL) & $817 \pm 49.2$ & $881 \pm 37.9$ & $803 \pm 53.8$ & $877 \pm 31.5$ \\
\hline
\end{tabular}

$\mathrm{SD}=$ standard diet; $\mathrm{F}=$ high fat diet; F-OSC =Opuntia streptacantha cladode powder; F-OFI = Opuntia ficus-indica cladode powder. AST $=$ aspartate aminotransferase. ALT = alanine aminotransferase. $\mathrm{MCP}-1$ = monocyte chemoattractant protein 1 . Results are mean of 10 animals \pm SEM. No significant difference were observed. energy demand. These cells represent a good model for investigating molecules able to reduce obesity via an impairment of differentiation and adipogenesis. In this work, we used the well-characterised murine preadipose 3 T3-F442A cell line for exploring the effects of OCP on adipogenesis $[29,30]$. Our results indicate that cladode powders from two different species effectively alter adipogenesis by reducing TG accumulation during the differentiation process, without generating cytotoxicity. This observation is in agreement with previous studies showing that OCP (powders or ethanol extracts) may reduce adipocyte differentiation and adipogenesis $[22,27,31,32]$. The differentiation of $3 \mathrm{~T} 3-\mathrm{F} 442 \mathrm{~A}$ preadipocytes in mature adipocytes by insulin includes an increase in both TG content and glucose uptake [33]. Our data show that high OCP concentrations inhibited the uptake of glucose elicited by insulin in differentiating cells. It is important to emphasize that this low glucose uptake was not associated with an increased TG storage, which would be indicative of insulin resistance. In contrast, the association of low TG content and low glucose uptake elicited by OCP in conditions of preadipocyte differentiation by insulin, supports an inhibitory effect of these agents on the differentiating process into mature adipocytes. Accordingly, it could be hypothesised that OCP and especially OFI treatment, may reduce adipocyte storage of TG and consequently adipocyte hypertrophy. Our data show that both OCP (with OFI being the most effective) exert anti-adipogenic effects in the 3 T3-F442A cell line, at concentrations effective against low-density lipoprotein oxidation, foam cell formation, and atherogenesis in apoE-knockout mice, and in cellular models for colon cancer studies in vitro [13, 14]. OCP effects could be compared with those of resveratrol, which exerts anti-obesity effects by inhibiting glucose utilisation in 3 T3-F442A cell line [34].

The chemical composition and the presence of phenolic compounds in the different Opuntia species has been previously reported [12]. Among the molecules identified in OCP, flavonoids, quercetin, kaempferols and isorhamnetin, could be implicated in weight loss $[15,16,22,35,36]$. Our previous studies indicated that the levels of flavonoids, quercetin, kaempferols and isorhamnetin detected by mass spectroscopy (LC-MS/MS), are higher in OFI than in OSC [12], which may explain the higher efficacy of this OCP in reducing HFDinduced weight gain. It is to note that low OCP concentrations were not active, possibly due to very low concentrations of the different phenolic compounds in the powders. These data are in agreement with studies reported by Lee et al. [16, 36], who showed that purified molecules (isorhamnetin or kampferol) may inhibit adipocyte differentiation and lipid accumulation. Kampferol blocked the phosphorylation of AKT and mTOR, acting 

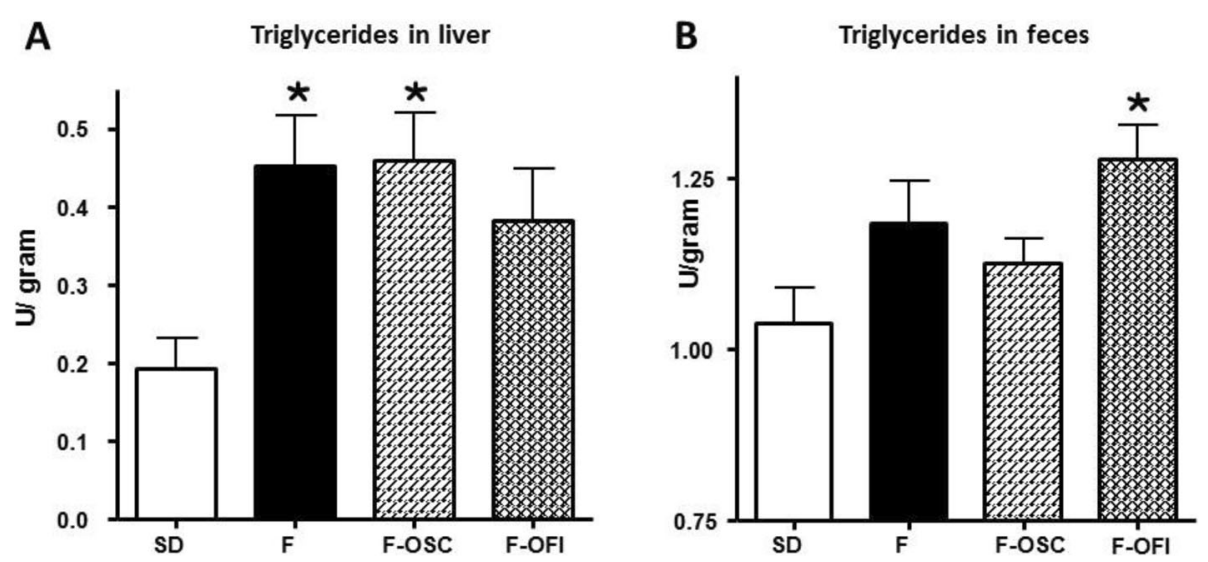

Fig. 5 Triglyceride content in liver (a) and faeces (b). Rats were fed with (F) or without (SD) a high-fat diet, supplemented with Opuntia cladode powders (OCP) from O. streptacanthaand O. ficus-indica, respectively (F-OSC, F-OFI). Triglyceride concentrations were reported relative to liver or faeces weight. Data represent mean \pm SEM. Statistical analysis involved ANOVA, followed by Newman-Keuls post hoc test. ${ }^{*} p<0.05$ indicates significant difference from group SD

on early adipogenic factors, which resulted in attenuation of late adipogenic factors such as C/EBP- $\alpha$ and PPAR $\gamma$. The same genes $(\mathrm{C} / \mathrm{EBP}-\alpha$ and PPAR $\gamma)$ and their target genes (LPL, aP2, LXR) were identified as isorhamnetin targets. Thus, these active compounds being present in Opuntia cladodes, they could act at the molecular level by regulating lipid metabolism. As OFI is the most domesticated cultivar, it could be of interest to select OFI species with higher phenolic compound content to improve their anti-obesity properties.

In our study, HFD-fed rats gained markedly more weight than those fed a normal diet, confirming that dietinduced obesity was successful. No noticeable difference was observed in food intake among all groups. Interestingly, OCP supplementation (F-OSC, F-OFI) prevented the weight of gain of animals with comparable food intake. Furthermore, OCP tended to reduce abdominal fat development over the 8 weeks of the diet without reducing food intake. Thus, we can conclude that OCP supplement in food could decrease the body weight gain by repressing the expansion of adipose tissue mass.

Leptin is a secreted peptide encoded by the obese gene and produced primarily by adipose cells. It plays a vital role in controlling body weight, presumably by acting in the hypothalamus to suppress appetite. Body fat is the most important determinant of circulating leptin levels, but other factors also acutely regulate the production and secretion of leptin, for instance, fasting decreases leptin, while refeeding restores the circulating leptin in both mice and humans [37]. Adiponectin is also secreted from adipocytes, and low circulating levels have been epidemiologically associated with obesity, insulin resistance, type 2 diabetes and cardiovascular diseases. Adiponectin promotes cell proliferation and differentiation of pre-adipocytes into adipocytes, augmenting programmed gene expression responsible for adipogenesis, and increasing lipid content and insulin responsiveness of the glucose transport system in adipocytes [38]. Circulating leptin levels are increased in HFD-fed animals, in parallel with a decrease in circulating adiponectin concentrations. In our study, serum adiponectin concentration was noticeably reduced in HFD-fed rats, which was reversed by OCP supplementation, suggesting that OCP treatment activated the adipocyte production of adiponectin. Moreover, serum leptin levels were increased in all HFD groups compared with the standard diet (SD), but the leptin levels were lower in the F-OSC and F-OFI groups. Leptin is known to regulate food intake and stimulates energy expenditure. As no differences were observed in food intake, the anti-obesity effects of OCP could be related to increased leptin sensitivity and modifications in energy expenditure. All these results are in agreement with an improvement in blood parameters related to obesity due to OCP supplementation in HFD. The same results were observed in a mouse model of diet-induced obesity, using isorhamnetin glycosides extracted from OFI [15], or using different bioactive compounds extracted from seaweed or ginseng leaf or Korean red ginseng on insulin sensitivity $[8,33]$.

A HFD is known to induce NAFLD in animal models and humans by causing fat deposition in the liver [39, 40]. NAFLD is closely associated with obesity. In our HFD-fed rat model of obesity, we showed that OCP supplement in the diet tends to lower the liver weight, which can be correlated to less TG storage in the liver. Similarly, Moran-Ramos et al. demonstrated that Opuntia cladode consumption attenuates hepatic steatosis in obese Zucker rats [18], and other studies based on HFD supplemented with quercetin [35] or Vignanakashimae extracts (another flavonoid-rich plant) [32] also resulted in a lowering of body weight gain 
and hepatic lipid accumulation. Taken together, these results suggest that OCP could be efficient against fatty liver in HFD obese rats. It is noteworthy that OCP treatment did not cause any detectable adverse toxic effects on the liver.

Uebelhack et al. [9] and Chong et al. [41] illustrated that the effects of Opuntia-derived fibers act in reducing dietary fat absorption in human volunteers, by binding to dietary fat and increasing its excretion in faeces, probably by decreasing fat intestinal absorption. To further understand the mechanisms explaining the lower weight gain induced by Opuntia supplementation in a HFD, we evaluated the faecal excretion of fats in our rat model, which was markedly increased in rats fed a HFD, and was much more pronounced when rats were supplemented with OFI. These findings support the hypothesis that the effects of OCP on weight are achieved by reducing dietary fat absorption, leading to lower energy intake and thus, to a lesser weight gain. Finally, nopal anti-inflammatory effects had been identified. Bouhini et al showed a reduction in low-grade chronic inflammation associated with obesity, this could be due to the effect of nopal fibers on the gut microbiota $[17,20]$.

\section{Conclusions}

Our study was performed using a rat model for obesity and a cellular model, which permits to reduce the number of animals used and obtain information about the cellular mode of action. However, using animals highlighted the decreased intestinal fat absorption and the non-toxic effects of dietary Opuntia powders, suggesting their potential to be used in the human diet. The mode of action of Opuntia in obesity management need to be further analyzed but it appears that some effects could be observed at the molecular level by regulating the adipocyte differentiation genes pathway, by reducing fat absorption as well as a possible modification of the gut microbiota. In conclusion, our data suggest that OFI cladode consumption may be useful in the management of obesity and the prevention of hepatic diseases (NAFLD) related to obesity.

\section{Abbreviations}

HFD: High-fat diet; NAFLD: Non-alcoholic fatty liver disease; OCP: Opuntia cladode powders; OFI: Opuntia ficus-indica; OSC: Opuntia streptacantha; TG: Triglycerides

\section{Acknowledgements}

The authors are grateful to Dr. Fabrice Pierre for helpful discussions and reviewing of the manuscript, and Prof. P. Valet (I2MC, Toulouse, France) for the cells.

\section{Authors' contributions}

$\mathrm{CHT}$ and FG conceived the idea and designed the experiments, analysed the data and wrote the manuscript. CHT and EF performed the experiments. NN and FBE assisted with animal management. MSSD and APBR provided the compounds. ANS contributed to cellular experiments and reading the manuscript. All authors reviewed the manuscript. All authors read and approved the final manuscript.

\section{Funding}

This work was supported by the bilateral French/Mexican ANR (French National Research Agency)/CONACYT (National Council of Science and Technology of Mexico) project BIOPUNTIA (ANR-2010-INTB-1702). It is academic funding. It had no role in the design of the study, interpretation of the results and writing of the manuscript.

\section{Availability of data and materials}

The datasets used and/or analysed during the current study are available from the corresponding author on reasonable request.

\section{Ethics approval}

The animals were from Charles Rivers Laboratory. The protocol was approved by the local ethics committee (TOXCOM/0011/FG FG). It follows the French "National Charter Concerning the Ethics of Animal Experimentation".

\section{Consent for publication}

Not applicable.

\section{Competing interests}

The authors declare that they have no competing interests.

\section{Author details}

${ }^{1}$ INRA, ToxAlim (Research Centre in Food Toxicology), Université de Toulouse, INRA, ENVT, INP-Purpan, UPS, Toulouse, France. ${ }^{2}$ Centro de Investigación y Estudios de Posgrado (CienciasQuímicas), UniversidadAutónoma de San Luis Potosí, San Luis Potosí, Mexico. ${ }^{3}$ Instituto Potosino de Investigación Científica y Tecnológica, San Luis Potosí, Mexico. ${ }^{4}$ INSERM U1048, Institute of Metabolic and Cardiovascular Diseases I2MC, Toulouse, France.

Received: 29 October 2019 Accepted: 20 January 2020

Published online: 05 February 2020

\section{References}

1. WHO | Obesity and overweight. WHO n.d. https://www.who.int/news-room/ fact-sheets/detail/obesity-and-overweight. Accessed 26 Oct 2017.

2. World Cancer Research Fund/American Institute for Cancer Research. Continuous Update Project Expert Report 2018. Diet, nutrition and physical activity: Energy balance and body fatness. 2018. Available at https://www.wcrf.org/ dietandcancer/energy-balance-body-fatness.

3. Avila-Nava A, Noriega LG, Tovar AR, Granados O, Perez-Cruz C, PedrazaChaverri J, et al. Food combination based on a pre-hispanic Mexican diet decreases metabolic and cognitive abnormalities and gut microbiota dysbiosis caused by a sucrose-enriched high-fat diet in rats. MolNutr Food Res. 2017;61. https://doi.org/10.1002/mnfr.201501023.

4. Rtveladze K, Marsh T, Barquera S, Romero LMS, Levy D, Melendez G, et al. Obesity prevalence in Mexico: impact on health and economic burden. Public Health Nutr. 2014;17:233-9. https://doi.org/10.1017/ S1368980013000086.

5. Hasani-Ranjbar S, Jouyandeh Z, Abdollahi M. A systematic review of antiobesity medicinal plants - an update. J Diabetes MetabDisord. 2013;12:28. https://doi.org/10.1186/2251-6581-12-28.

6. del Socorro Santos Díaz M, Barba de la Rosa A-P, Héliès-Toussaint C, Guéraud F, Nègre-Salvayre A. Opuntia spp.: Characterization and Benefits in Chronic Diseases. Oxidative Med Cell Longev. 2017. https://doi.org/10.1155/ $2017 / 8634249$.

7. Ojulari OV, Lee SG, Nam J-O. Beneficial Effects of Natural Bioactive Compounds from Hibiscus sabdariffa L on Obesity. Molecules. 2019;24:210. https://doi.org/10.3390/molecules24010210.

8. Yang H-W, Fernando KHN, Oh J-Y, Li X, Jeon Y-J, Ryu B. Anti-obesity and anti-diabetic effects of Ishigeokamurae. Mar Drugs. 2019;17:202. https://doi. org/10.3390/md17040202.

9. Uebelhack R, Busch R, Alt F, Beah Z-M, Chong P-W. Effects of Cactus Fiber on the excretion of dietary fat in healthy subjects: a double blind, randomized, placebo-controlled, crossover clinical investigation. Curr Ther Res. 2014;76:39-44. https://doi.org/10.1016/j.curtheres.2014.02.001.

10. Grube B, Chong P-W, Lau K-Z, Orzechowski H-D. A natural Fiber complex reduces body weight in the overweight and obese: a double-blind, randomized, Placebo-Controlled Study. Obesity. 2012. https://doi.org/10. 1038/oby.2012.165. 
11. El-Mostafa K, Kharrassi Y, Badreddine A, Andreoletti P, Vamecq J, Kebbaj M, et al. Nopal Cactus (Opuntiaficus-indica) as a source of bioactive compounds for nutrition, health and disease. Molecules. 2014;19:14879-901. https://doi.org/10.3390/molecules190914879.

12. Astello-García MG, Cervantes I, Nair V. Santos-Díaz M del S, Reyes-Agüero a, Guéraud $F$, et al. chemical composition and phenolic compounds profile of cladodes from Opuntia spp. cultivars with different domestication gradient. J Food Compos Anal. 2015;43:119-30. https://doi.org/10.1016/j.jfca.2015.04.016.

13. Keller J, Camaré C, Bernis C, Astello-García M, de la Rosa A-PB, Rossignol M, et al. Antiatherogenic and antitumoral properties of Opuntia cladodes: inhibition of low density lipoprotein oxidation by vascular cells, and protection against the cytotoxicity of lipid oxidation product 4hydroxynonenal in a colorectal cancer cellular model. J Physiol Biochem. 2015;71:577-87. https://doi.org/10.1007/s13105-015-0408-x.

14. Garoby-Salom S, Guéraud F, Camaré $C$, de la Rosa A-PB, Rossignol M, del SS $\mathrm{DM}$, et al. Dietary cladode powder from wild type and domesticated Opuntia species reduces atherogenesis in apoEknock-out mice. J Physiol Biochem. 2016;72:59-70.

15. Rodríguez-Rodríguez C, Torres N, Gutiérrez-Uribe JA, Noriega LG, TorreVillalvazo I, Leal-Díaz AM, et al. The effect of isorhamnetin glycosides extracted from Opuntiaficus-indica in a mouse model of diet induced obesity. Food Funct. 2015;6:805-15. https://doi.org/10.1039/C4FO01092B.

16. Lee Y-J, Choi H-S, Seo M-J, Jeon H-J, Kim K-J, Lee B-Y. Kaempferol suppresses lipid accumulation by inhibiting early adipogenesis in 3T3-L1 cells and zebrafish. Food Funct. 2015;6:2824-33. https://doi.org/10.1039/C5FO00481K.

17. Sánchez-Tapia M, Aguilar-López M, Pérez-Cruz C, Pichardo-Ontiveros E, Wang M, Donovan SM, et al. Nopal (Opuntiaficusindica) protects from metabolic endotoxemia by modifying gut microbiota in obese rats fed high fat/sucrose diet. Sci Rep. 2017;7. https://doi.org/10.1038/s41598-017-05096-4

18. Morán-Ramos S, Avila-Nava A, Tovar AR, Pedraza-Chaverri J, López-Romero P, Torres N. Opuntiaficusindica (nopal) attenuates hepatic steatosis and oxidative stress in obese Zucker (fa/fa) rats. J Nutr. 2012;142:1956-63. https://doi.org/10.3945/jn.112.165563.

19. Zang $Y$, Zhang $L$, lgarashi $K$, Yu C. The anti-obesity and anti-diabetic effects of kaempferol glycosides from unripe soybean leaves in high-fat-diet mice. Food Funct. 2015;6:834-41. https://doi.org/10.1039/c4fo00844h.

20. Bounihi A, Bitam A, Bouazza A, Yargui L, Koceir EA. Fruit vinegars attenuate cardiac injury via anti-inflammatory and anti-adiposity actions in high-fat diet-induced obese rats. Pharm Biol. 2017:55:43-52. https://doi.org/10.1080/ 13880209.2016.1226369.

21. Lee SG, Lee YJ, Jang M-H, Kwon TR, Nam J-O. Panax ginseng leaf extracts exert anti-obesity effects in high-fat diet-induced obese rats. Nutrients. 2017; 9:999. https://doi.org/10.3390/nu9090999.

22. Moon J, Do H-J, Kim OY, Shin M-J. Antiobesity effects of quercetin-rich onion peel extract on the differentiation of 3T3-L1 preadipocytes and the adipogenesis in high fat-fed rats. Food Chem Toxicol. 2013;58:347-54. https://doi.org/10.1016/j.fct.2013.05.006.

23. Ramírez-Tobías HM, Reyes-Agüero JA, Pinos-Rodríguez JM, Aguirre-Rivera JR, Ramírez-Tobías HM, Reyes-Agüero JA, et al. Efecto de la especie y madurez sobre el contenido de nutrientes de cladodios de nopal. Agrociencia. 2007:41:619-26.

24. Kim JH, Park JM, Kim E-K, Lee JO, Lee SK, Jung JH, et al. Curcumin stimulates glucose uptake through AMPK-p38 MAPK pathways in L6 myotube cells. J Cell Physiol. 2010:n/a-n/a. https://doi.org/10.1002/jcp.22093.

25. Héliès-Toussaint C, Peyre L, Costanzo C, Chagnon M-C, Rahmani R. Is bisphenol $\mathrm{S}$ a safe substitute for bisphenol a in terms of metabolic function? An in vitro study. Toxicol Appl Pharmacol. 2014;280:224-35. https://doi.org/10.1016/j.taap.2014.07.025.

26. Rosen ED, Spiegelman BM. Adipocytes as regulators of energy balance and glucose homeostasis. Nature. 2006:444:847-53. https:/doi.org/10.1038/nature05483.

27. Lai C-S, Liao S-N, Tsai M-L, Kalyanam N, Majeed M, Majeed A, et al. Calebin-a inhibits adipogenesis and hepatic steatosis in high-fat diet-induced obesity via activation of AMPK signaling. Mol Nutr Food Res. 2015;59:1883-95. https://doi.org/10.1002/mnfr.201400809.

28. Jensen VS, Hvid H, Damgaard J, Nygaard H, Ingvorsen C, Wulff EM, et al. Dietary fat stimulates development of NAFLD more potently than dietary fructose in Sprague-Dawley rats. DiabetolMetabSyndr. 2018;10:4. https://doi. org/10.1186/s13098-018-0307-8

29. Alonso-Castro AJ, Salazar-Olivo LA. The anti-diabetic properties of Guazumaulmifolia lam are mediated by the stimulation of glucose uptake in normal and diabetic adipocytes without inducing adipogenesis. J Ethnopharmacol. 2008;118:252-6. https://doi.org/10.1016/j.jep.2008.04.007.
30. Alonso-Castro AJ, Zapata-Bustos R, Romo-Yañez J, Camarillo-Ledesma P, Gómez-Sánchez M, Salazar-Olivo LA. The antidiabetic plants Tecomastans (L. ) Juss. exKunth (Bignoniaceae) and Teucriumcubense Jacq (Lamiaceae) induce the incorporation of glucose in insulin-sensitive and insulin-resistant murine and human adipocytes. J Ethnopharmacol. 2010;127:1-6. https://doi. org/10.1016/j.jep.2009.09.060.

31. Song Y, Park HJ, Kang SN, Jang S-H, Lee S-J, Ko Y-G, et al. Blueberry Peel extracts inhibit Adipogenesis in 3T3-L1 cells and reduce high-fat dietinduced obesity. PLoS One. 2013;8:e69925. https://doi.org/10.1371/journal. pone.0069925.

32. Son $Y$, Nam J-S, Jang M-K, Jung I-A, Cho S-I, Jung M-H. Antiobesity activity of Vignanakashimae extract in high-fat diet-induced obesity. Biosci Biotechnol Biochem. 2013;77:332-8. https://doi.org/10.1271/bbb.120755.

33. Yu Z-W, Burén J, Enerbäck S, Nilsson E, Samuelsson L, Eriksson JW. Insulin can enhance GLUT4 gene expression in 3T3-F442A cells and this effect is mimicked by vanadate but counteracted by CAMP and high glucose potential implications for insulin resistance. Biochimica et BiophysicaActa (BBA) - Molecular Basis of Disease. 2001;1535:174-85. https://doi.org/10. 1016/S0925-4439(00)00097-1.

34. Carpéné C, Les F, Cásedas G, Peiro C, Fontaine J, Chaplin A, et al. Resveratrol anti-obesity effects: rapid inhibition of adipocyte glucose utilization. Antioxidants. 2019:8:74. https://doi.org/10.3390/antiox8030074.

35. Hoek-van den Hil EF, van Schothorst EM, van der Stelt I, Swarts HJM, Venema D, Sailer M, et al. Quercetin decreases high-fat diet induced body weight gain and accumulation of hepatic and circulating lipids in mice. Genes Nutr. 2014:9. https://doi.org/10.1007/s12263-014-0418-2.

36. Lee J, Jung E, Lee J, Kim S, Huh S, Kim Y, et al. Isorhamnetin represses Adipogenesis in 3T3-L1 cells. Obesity. 2009;17:226-32. https://doi.org/10. 1038/oby.2008.472.

37. Barr VA, Malide D, Zarnowski MJ, Taylor SI, Cushman SW. Insulin stimulates both leptin secretion and production by rat white adipose tissue. Endocrinology. 1997;138:4463-72.

38. Fu Y, Luo N, Klein RL, Garvey WT. Adiponectin promotes adipocyte differentiation, insulin sensitivity, and lipid accumulation. J Lipid Res. 2005; 46:1369-79. https://doi.org/10.1194/jlr. M400373-JLR200.

39. Kim M-H, Kang K-S, Lee Y-S. The inhibitory effect of genistein on hepatic steatosis is linked to visceral adipocyte metabolism in mice with dietinduced non-alcoholic fatty liver disease. Br J Nutr. 2010;104:1333-42. https://doi.org/10.1017/S0007114510002266.

40. Anderson N, Borlak J. Molecular mechanisms and therapeutic targets in Steatosis and Steatohepatitis. Pharmacol Rev. 2008;60:311-57. https://doi. org/10.1124/pr.108.00001.

41. Chong P-W, Lau K-Z, Gruenwald J, Uebelhack R. A review of the efficacy and safety of Litramine IQP-G-002AS, an Opuntiaficus-indica derived Fiber for weight management. Evid Based Complement Alternat Med. 2014;2014:1-6. https://doi.org/10.1155/2014/943713.

\section{Publisher's Note}

Springer Nature remains neutral with regard to jurisdictional claims in published maps and institutional affiliations.

Ready to submit your research? Choose BMC and benefit from

- fast, convenient online submission

- thorough peer review by experienced researchers in your field

- rapid publication on acceptance

- support for research data, including large and complex data types

- gold Open Access which fosters wider collaboration and increased citations

- maximum visibility for your research: over $100 \mathrm{M}$ website views per year

At $\mathrm{BMC}$, research is always in progress.

Learn more biomedcentral.com/submission 